\title{
Density profile asymptotes at the centre of dark matter halos
}

\author{
J. P. Mücket ${ }^{1}$ and M. Hoeft ${ }^{2}$ \\ 1 Astrophysikalisches Institut Potsdam, An der Sternwarte 16, 14482 Potsdam, Germany \\ 2 International University Bremen, Campusring 1, 28759 Bremen, Germany \\ e-mail: m.hoeft@iu-bremen.de
}

Received 21 January 2003 / Accepted 10 April 2003

\begin{abstract}
For the spherical symmetric case, all quantities describing the relaxed dark matter halo can be expressed as functions of the gravitational potential $\Phi$. Decomposing the radial velocity dispersion $\sigma_{\mathrm{r}}$ with respect to $\Phi$ at very large and very small radial distances the possible asymptotic behavior for the density and velocity profiles can be obtained. If reasonable boundary conditions are posed such as a finite halo mass and force-free halo centre the asymptotic density profiles at the centre should be much less steep than the profiles obtained within numerical simulations. In particular cases profiles like Plummer's model are obtained. The reasons of that seeming discrepancy with respect to the results of $N$-body simulations are discussed.
\end{abstract}

Key words. cosmology: theory - dark matter - galaxies: formation - galaxies: structure - methods: analytical

\section{Introduction}

The formation of structures by gravitational interacting cold dark matter $(\mathrm{CDM})$ is one of the outstanding paradigms in cosmology. The luminous baryonic matter is embedded in dark matter halos. Numerical studies of the structure formation, which allows only for gravitational interactions, predict the distribution of galaxies and clusters of galaxies in excellent agreement with observations. Special attention has been devoted to the investigation of halos as typical aggregates forming after collapse and violent relaxation.

Early studies seemed to indicate that not only the distribution of halos but also their density profiles depend on the underlying cosmological model (Quinn et al. 1986; Frenk et al. 1988; Dubinski \& Carlberg 1991; Crone et al. 1994). However, numerous numerical investigations have shown that halos have an almost universal profile that is independent of the mass of the halo (Navarro et al. 1996, 1997), the initial fluctuation spectrum and the underlying cosmological model. Although the resolution of the simulations has increased dramatically since the first studies, high resolution simulations are still not able to provide a reliable determination of the shape of the innermost density profiles. Navarro et al. $(1996,1997)$ first proposed an analytic approximation for the density profiles obtained in simulations. They obtained $\rho \propto\left(r / r_{\mathrm{s}}\right)^{-1}\left(1+r / r_{\mathrm{s}}\right)^{-2}$, where $r_{\mathrm{s}}$ denotes the scale radius of a given halo. This NFW-profile implies an asymptotic behavior $\rho \propto r^{-n}$ with a power index of $n=1$ for the density profile throughout the central halo region. Other numerical studies produced a significantly steeper inner slope

Send offprint requests to: J. P. Mücket,

e-mail: jpmuecket@aip.de corresponding to $n \approx 1.5$ (Moore et al. 1998, 1999; Ghigna et al. 1998, 2000; Fukushige \& Makino 2001; Klypin et al. 2001). In contrast to these results Power et al. (2003) did not find an asymptotic power behavior with fixed index at all but rather a continuously decreasing index with values down to $\approx 1.2$ at the innermost radius they could resolve. Finally, Jing $\&$ Suto (2000) claimed that the asymptotic behavior of the innermost region depends on the mass of the halo.

The observations yield a different picture: From the rotation curves of stars the density profile of the underlying dark matter halo of a galaxy can be inferred. The observation that Low Surface Brightness (LSB) and High Surface Brightness (HSB) galaxies follow the same Tully-Fisher relation requires (in the conventional picture) that LSB galaxies are dominated by dark matter (DM), e.g., Verheijen (1997), de Blok \& McGaugh (1996). They are promising candidates for determining the innermost density profile. A "cuspy", $n \geq 1$, dark matter core is ruled out by many studies (McGaugh \& de Blok 1998; de Blok et al. 2001). It is also ruled out by modeling the inner rotation curve of the Galaxy (Binney \& Evans 2001). In contrast to this results some recent investigations of dwarf galaxies suggest that an innermost slope of $n \approx 1$ is consistent with rotation curve data (van den Bosch \& Swaters 2001). Thus, present observations permit an inner slope of $n \leq 1$ at most. El-Zant et al. (2001) suggest that a "cuspy" halo core may be eliminated by dynamical friction of an initially clumpy gas distribution. This indicates that the gas content of a halo may affect the dark matter profile.

Taylor \& Navarro (2001) argued that the recurrent merging process results in a phase-space density profile which decreases according to a power-law. With $\rho / \sigma^{3} \propto r^{-1.875}$ they reproduced 
well the numerical density profiles. Navarro (2001) argued that the entropy in a halo is most uniform distributed under the condition of a power-law phase-space density. This results in observed density profiles. In contrast to an universal density profile some authors predict again a dependency on the formation history and the underlying cosmological model (Syer \& White 1998; Nusser \& Sheth 1999; Łokas \& Hoffman 2000). Recent papers by Dekel et al. (2002), Weinberg \& Katz (2002) and Sellwood (2003) discuss the influence of subsequent mergers or central bars onto the formation of cusps at the dark matter halo centre. Though the discussion about the bar influence is still controversial the common outcome of those investigations is that a cusp seems not to be a generic feature of a relaxed dark matter halo but is rather the result of further interaction with the halo surroundings. The effect on the halo profiles due to the interaction between the gas and the dark matter on the profiles is still an open question. The possible structure of pure dark matter halos is still a basic problem and needs to be considered separately to get insight into the more general behavior of the combined system of dark matter and gas.

In this paper we suppose that the halos have spherical symmetry and are sufficiently well relaxed, i.e., the halos can be described by the steady state Jeans equation. We systematically investigate the asymptotic behavior of the quantities describing the halo at radii comparable to and larger than the virial radius (outer region) and near the central region at small radii. The assumptions to be made will be kept very general, e.g., demanding the finiteness of the halo mass.

The most essential and probably the most restrictive boundary condition we will adopt is the demand for a nonsingular potential throughout. This appears as an appropriate assumption for real dark matter halos if the existence of a central point mass is forbidden.

A particular aim of the present paper is to investigate under which conditions a power asymptote with respect to the radius exists for the density profiles of dark matter halos if approaching the very centre.

\section{The asymptotes at large radii}

The system of equations describing spherically symmetric halo configurations consisting of collisionless dark matter particles is formed by the Jeans equation and the Poisson equation. The halo structure is completely described by the density $\rho(r)$, the components of the velocity dispersion $\sigma^{2}=\sigma_{\mathrm{r}}^{2}+\sigma_{\theta}^{2}+\sigma_{\phi}^{2}$ and the gravitational potential $\Phi(r)$. Due to spherical symmetry it follows $\sigma_{\theta}^{2}=\sigma_{\phi}^{2}$. In order to close up the above system of equations one needs two more relations. Even if supposing a nearly constant anisotropy parameter $\beta=1-\sigma_{\theta}^{2} / \sigma_{\mathrm{r}}^{2}$ still a further relation is needed. Asymptotic considerations may provide such a relation from some basic assumptions.

For the spherical-symmetric case, the potential $\Phi(r)$ is an increasing monotonic function with respect to the radius $r$; $\mathrm{d} \Phi(r) / \mathrm{d} r=G M(r) / r^{2}>0$ at $r>0$, where $M(r)$ is the cumulative mass at radius $r$. Therefore, all quantities can be expressed as functions of $\Phi$ instead of $r$. The potential $\Phi$ is determined up to an arbitrary constant which has to be fixed by the chosen boundary conditions usually given either at $r \rightarrow \infty$ or at $r=0$ or at a finite boundary $r=R$ if it exists for the considered object. We suppose a non-singular potential throughout. Then it can be recalibrated to fulfill $\Phi(0)=0$ at the center of the halos. Further, we assume the potential to be sufficiently smooth to fulfill the boundary condition for $\mathrm{d} \Phi / \mathrm{d} r$ at $r=0$

$\frac{\mathrm{d} \Phi}{\mathrm{d} r}(0)=0$

which we adopt throughout.

We suppose that the entire mass of the halo is finite.

The latter condition means that at $r \rightarrow 0$ the density $\rho$ must not increase faster than $\rho \propto r^{\epsilon-3}$ where $\epsilon>0$ can take an arbitrary small value. The condition (1) requires that the density $\rho$ at $r \rightarrow 0$ must not increase faster than $\rho(r) \propto r^{-1+\epsilon}$ with $\epsilon>0$. Otherwise $\mathrm{d} \Phi / \mathrm{d} r=M(r) / r^{2}$ becomes singular at $r \rightarrow 0$. Thus the condition (1) leads to an even stronger restriction for the density behavior fulfilling the supposition of finite halo mass at $r \rightarrow 0$ automatically.

At $r \rightarrow \infty$ the potential $\Phi(r)$ approaches $\Phi_{\infty}$. Since at $r \rightarrow \infty$ the probability to find particles with nonzero velocities should vanish, it follows also $\sigma_{\mathrm{r}}^{2} \rightarrow 0$ at $r \rightarrow \infty$. A decomposition of $\sigma_{\mathrm{r}}^{2}$ into a power series in the vicinity of $\Phi_{\infty}$ can be achieved, where the leading term is $\propto\left(\Phi_{\infty}-\Phi\right)^{p}$ with $p>0$.

Therefore, $\sigma_{\mathrm{r}}^{2}$ can be represented in terms

$\sigma_{\mathrm{r}}^{2}=\alpha\left(\Phi_{\infty}-\Phi\right)^{p}=\alpha \Psi^{p}(r)$,

where $\alpha$ is a positive constant which has to be determined. Here we adopted the so-called relative potential (see e.g. Binney \& Tremaine 1987) $\Psi=\Phi_{\infty}-\Phi(r)$.

In this case the Jeans equation

$\frac{\mathrm{d}\left(\rho \sigma_{\mathrm{r}}^{2}\right)}{\mathrm{d} r}+2 \beta \frac{\rho}{r} \sigma_{\mathrm{r}}^{2}=-\rho \frac{\mathrm{d} \Phi}{\mathrm{d} r}$

can be integrated and we obtain

$\rho \propto \Psi^{-p} r^{-2 \beta} \exp \left(\frac{\Psi^{1-p}}{\alpha(1-p)}\right)$.

In addition to a finite halo mass the density must vanish $\rho \rightarrow 0$ at $r \rightarrow \infty$. Although the asymptotic behavior at $\Psi \rightarrow 0$ is different for $p<1$ from the behavior for $p>1$ this must be satisfied by all possible $p$. The singular case $p=1$ must be considered separately. If $p<1$ the asymptotic behavior of $\rho(r)$ at $\Psi \rightarrow 0$ is determined by the terms in front of the exponential term

$\rho \propto \Psi^{-p} r^{-2 \beta}$

Inserting (5) into the Poisson equation

$\frac{1}{r^{2}} \frac{\mathrm{d}}{\mathrm{d} r}\left(r^{2} \frac{\mathrm{d} \Psi}{\mathrm{d} r}\right)=4 \pi G \rho$,

we get

$\frac{1}{r^{2}} \frac{\mathrm{d}}{\mathrm{d} r}\left(r^{2} \frac{\mathrm{d} \Psi}{\mathrm{d} r}\right)+4 \pi G c \Psi^{-p} r^{-2 \beta}=0$.

One solution of Eq. (7) is a power law with respect to $r$ which gives at the same time the asymptote for large $r$ where the density $\rho$ must be a decreasing function. 
Assuming $\Psi \propto r^{l}$ a relation between the exponent $l$ and the parameters $\beta$ and $p$ introduced above can be given:

$l=\frac{2(1-\beta)}{1+p}$.

Since always $\beta \leq 1$ it follows from (8) that Eq. (7) does not permit a power asymptote which fulfills the condition $\Psi(r) \rightarrow$ 0 at $r \rightarrow \infty$.

For the cases $p>1$ according to (4) the density goes exponentially fast to zero for $\Psi \rightarrow 0$ at $r \rightarrow \infty$. Those models were obtained, e.g., by Milgrom (1996). This means that the density term in the Poisson equation vanishes much faster than the first two terms $\mathrm{d}^{2} \Psi / \mathrm{d} r^{2}+(2 / r) \mathrm{d} \Psi / \mathrm{d} r$. Hence the asymptotic solution for $\Psi$ is determined just by these terms and we obtain $\Psi(r) \propto 1 / r$. The solution with exponential decrease of the density at $r \rightarrow \infty$ describes a halo the mass of which is distributed within an finite radius $R_{\text {eff }}$ and the asymptotic solution for $\Psi$ is equivalent to the potential describing the outer region at $r>R_{\text {eff }}$ approaching the outer solution for a spherical symmetric finite body. The radius $R_{\text {eff }}$ is determined by the potential value when the exponent in (4) becomes effective. Provided the halo mass $M_{0}$ is known then the halo radius can be estimated to be $R_{\text {eff }} \approx G M_{0}(\alpha(p-1))^{1 /(p-1)}$.

Now, let us consider the particular case $p=1$. In this case, the Jeans equation can be integrated neglecting initially a possible anisotropy of the velocity dispersion. We find

$\rho=c \Psi^{\gamma}$

where $\gamma=(1-\alpha) / \alpha$. We use Eq. (9) to eliminate $\rho$ from Poisson's equation and find

$\frac{1}{r^{2}} \frac{\mathrm{d}}{\mathrm{d} r}\left(r^{2} \frac{\mathrm{d} \Psi}{\mathrm{d} r}\right)+4 \pi G c \Psi^{\gamma}=0$,

which is known as Lane-Emden equation. One solution of Eq. (10) is a power law with respect to $r$

$\Psi \propto r^{2 \alpha /(2 \alpha-1)}$.

Assuming $\rho \propto r^{n}$, a relation between the exponent $n$ and the coefficient $\alpha$ can be obtained:

$n=\frac{2(1-\alpha)}{2 \alpha-1}$.

Since $\alpha>0$ and $n<0$ we get either

$0<\alpha<\frac{1}{2}$

or

$\alpha>1$.

The latter condition can be excluded since it leads to infinite halo masses. The condition $0<\alpha<\frac{1}{2}$ satisfies the boundary condition $\Psi(r) \rightarrow 0$ at $r \rightarrow \infty$, at the same time.

The demand of a finite halo mass will be satisfied if $n<-3$. This restricts $\alpha$ further via

$\frac{1}{4}<\alpha<\frac{1}{2}$.
The special case of $\alpha=1 / 4$ results in the asymptotic behavior of the NFW profile.

If we admit anisotropy for the velocity dispersion which is indicated by the results of the halo analysis from $N$-body simulations for the outer region then we have to consider the Jeans equation with $\beta \neq 0$.

Assuming $\beta \approx$ const. and using the relation (2) we are again able to integrate (3) obtaining

$\rho=c \frac{\Psi^{\gamma}}{r^{2 \beta}}$

The asymptotic power laws for $\Psi$ and $\rho$ are then $\Psi \propto r^{n}$ with $n=2(1-\beta) /(1-\gamma)$ and $\rho \propto r^{m}$ with $m=n \gamma-2 \beta=2(\gamma-\beta) /(1-$ $\gamma)$. Arguments as given above lead to the condition for $\alpha$

$\frac{1}{4-2 \beta}<\alpha<\frac{1}{2}$

For $\beta=0$, the above obtained results are recovered. The case $\beta=1$ must be considered separately. We solve the Jeans equation for this case and obtain that $\alpha=1 / 2$ is necessary for the existence of a power asymptote. The finite-mass condition rules out this asymptote, however.

The case $\alpha=1 / 2$ is also not included within the above considerations. For an arbitrary $\beta \neq 1$ this leads to the asymptotes of Bessel functions at $r \rightarrow \infty$ also not fulfilling the finite-masscondition.

Thus, an anisotropy with respect to the velocity dispersion $\beta \neq 0$ confines the possible range for $\alpha$, i.e., this leads to the more restrictive condition (17).

\section{The asymptotes at the innermost halo region}

We shall now consider the central part of the halos in more detail. The knowledge about this region is uncertain partly because of the lack of resolution and also due to the poor particle statistics when approaching $r \rightarrow 0$. So the question whether the solution approaches a power asymptote within the central region or not will be considered. Provided a power asymptote exists then its possible properties will be analyzed.

As was argued above for the spherical-symmetric case all quantities could be represented as functions of the potential $\Phi(r)$. For the above adopted boundary conditions $(\Phi(0)=$ $0)$ we consider the decomposition of the velocity dispersion into a power series with respect to $\Phi$. In order to allow for the case that $\sigma_{\mathrm{r}}$ exhibits a power-like behavior with an arbitrary exponent at $\Phi=0$ we consider the more general decomposition

$\sigma_{\mathrm{r}}^{2}=C_{0} \Phi^{\kappa}\left(1+a_{1} \Phi+a_{2} \Phi^{2} \ldots\right)$

We first consider the case $\kappa \neq 0$. Then the leading term at $r \rightarrow 0$ is

$\sigma_{\mathrm{r}}^{2}=C_{0} \Phi^{\kappa}$

where $\kappa>0$ if supposing regularity of $\sigma^{2}(r \rightarrow 0)$. Neglecting anisotropy, we get from the Jeans equation:

$\rho \propto \Phi^{-\kappa} \exp \left(-\frac{\Phi^{1-\kappa}}{C_{0}(1-\kappa)}\right)$ 


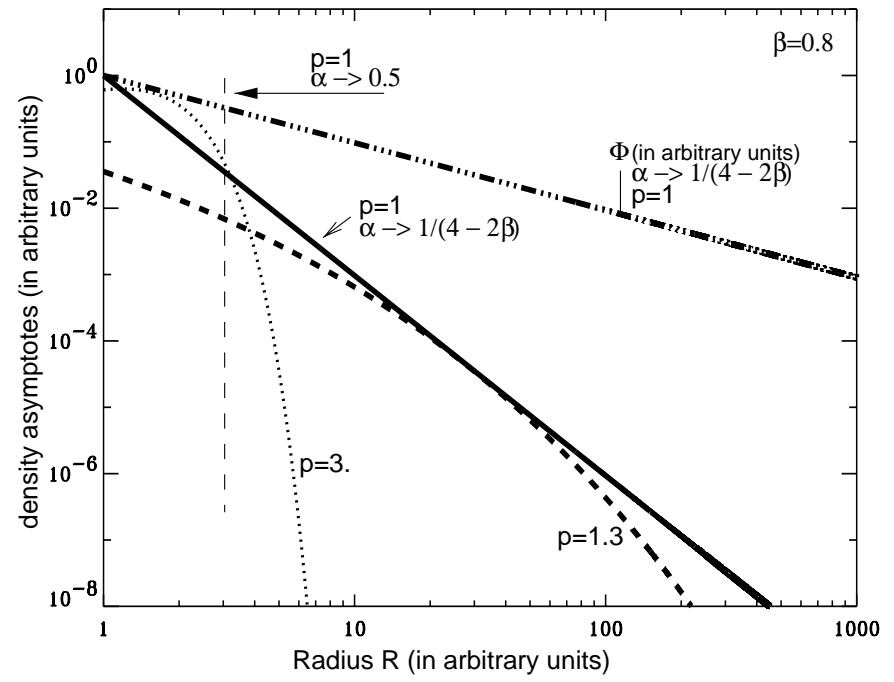

Fig. 1. The figure shows the shapes of the obtained asymptotes at $r \rightarrow$ $\infty$ covering the area between the asymptote with $(p=1, \alpha \rightarrow 1 /(4-$ $2 \beta)$ ) (thick solid line) and the dashed vertical line $(p=1, \alpha \rightarrow 1 / 2)$. For comparison two asymptotes for $p>1$ are shown: The asymptote with $p=1.3$ approaches for decreasing $p$ the $(p=1, \alpha \rightarrow 1 /(4-$ $2 \beta$ ) asymptote (thick dashed line), the $p=3$ asymptote exhibits an extremely steep decrease (dotted line). Asymptotes with increasing $p$ approach the vertical dashed line. For $p>1$ the density falls off very rapidly after some characteristic radius and describes a finite halo. All asymptotes are given for $\beta=0.8$.

In order to avoid an exponential-like singularity of the density at $r \rightarrow 0$ for $\kappa$ must be valid $\kappa<1$. The case $\kappa>1$ would also lead to an infinite halo mass as long $\Phi$ can be represented as some power of $r$ at $r \rightarrow 0$. Although we will restrict ourselves on just this case in some considerations below, we should be are aware of this is an additional assumption. Therefore, the density behavior near the center is given by

$\rho \approx \Phi^{-\kappa}$

where $\Phi \rightarrow 0$.

This is true as long as the exponent in (20) is small with respect to unity, i.e., $\Phi \ll\left(C_{0}(1-\kappa)\right)^{1 /(1-\kappa)}$. The potential $\Phi$ can be scaled with respect to its value $\Phi_{\infty}$ at $r \rightarrow \infty$. Then $C_{0}=$ $c \Phi_{\infty}^{1-\kappa}$ where the comparison with the results of high-resolution numerical $N$-body simulations (see Hoeft et al. 2003) provides a numerical value of about $c \approx 0.3$.

Inserting Eq. (21) into the Poisson equation we can find the asymptotic solutions for $\Phi$ at $r \rightarrow 0$. Due to nonlinearity several kinds of solutions are possible, in principle. If we restrict ourselves to solutions for $\Phi$ having a power-law behavior with respect to $r$ near $r=0$ then we get for the asymptotic behavior

$\Phi \propto r^{2 /(1+\kappa)}$

$\sigma_{\mathrm{r}}^{2} \propto \Phi^{\kappa} \propto r^{2 \kappa /(1+\kappa)}$.

Though $\rho \propto \Phi^{-\kappa} \propto r^{-2 \kappa /(1+\kappa)}$ is singular at $r \rightarrow 0$ the halo mass remains always finite if $0<\kappa<1$.

The case $\kappa=1$ must be considered as a special case solving Eq. (19) and leads to a separate solution not covered by (20)

$\rho \propto \Phi^{-\left(\frac{1+C_{0}}{C_{0}}\right)} \propto r^{-2\left(1+C_{0}\right) /\left(2 C_{0}+1\right)}$.
From (1) follows the condition

$2\left(1+C_{0}\right) /\left(2 C_{0}+1\right)<1$

which cannot be satisfied for any possible $C_{0}$. Therefore the case $\kappa=1$ is ruled out.

An almost isotropic velocity dispersion is justified at $r \rightarrow 0$ by the results of numerical simulations. In general $\beta$ is a function of $r$, which is small or vanishes if $r \rightarrow 0$. From the simulation results, it is known that $\beta(r)$ is an increasing function of the radial coordinate. If at $r \rightarrow 0 \beta$ is non-zero, i.e., $\beta \rightarrow \beta_{0}$ at $r \rightarrow 0$, then

$\rho \propto r^{-2 \beta_{0}} \Phi^{-\kappa} \exp \left(-\frac{\Phi^{1-\kappa}}{C_{0}(1-\kappa)}\right)$

$\Phi \propto r^{2\left(1-\beta_{0}\right) /(1+\kappa)}$

$\sigma_{\mathrm{r}}^{2} \propto \Phi^{\kappa} \propto r^{2\left(1-\beta_{0}\right) \kappa /(1+\kappa)}$.

For $\beta \rightarrow 0$ at $r \rightarrow 0$ we can make a power law ansatz $\beta \propto r^{\mu}$ with $\mu>0$. This leads to a solution for $\rho$ similar to the expression (20) containing in the exponent an additive term proportional to $-r^{\mu}$ which leads at $r \rightarrow 0$ again to the case (21). If we pose the additional condition that the term $\mathrm{d}\left(\rho \sigma^{2}\right) / \mathrm{d} r$ being the analog for the pressure gradient should vanish or should be regular, at least, at $r \rightarrow 0$ then it follows immediately $\beta(r \rightarrow 0) \rightarrow 0$, i.e., also $\beta_{0}=0$, which is consistent with other considerations.

We use the relation $\mathrm{d}\left(\rho \sigma^{2}\right) / \mathrm{d} r=-\rho \mathrm{d} \Phi / \mathrm{d} r$ and suppose that $\rho$ is a power law with respect to $r$ and obtain

$\frac{\mathrm{d}\left(\rho \sigma^{2}\right)}{\mathrm{d} r} \propto r^{(-3 \kappa+1) /(1+k)}$

Note, in order to obtain the correct expression being consistent with the right-hand side of the Jeans equation one has to perform first the derivative $\mathrm{d}\left(\rho \sigma^{2}\right) / \mathrm{d} r$ using the expression (20) and considering the limit $\Phi \rightarrow 0$ only afterwards.

Regularity of $\mathrm{d}\left(\rho \sigma^{2}\right) / \mathrm{d} r$ at $r \rightarrow 0$ leads immediately to the condition $0<\kappa \leq 1 / 3$. Thus the behavior of $\rho$ at $r \approx 0$ is given by $\rho \propto r^{-m}$ with a possible range for $0<m \leq 1 / 2$.

This result is a consequence of demanding the right-hand side of the Jeans equation to be non-singular at $r \rightarrow 0$, i.e., requiring not only $\mathrm{d} \Phi / \mathrm{d} r=0$ but also the product of $\rho \mathrm{d} \Phi / \mathrm{d} r$ to be finite, at least. This leads inevitably to a further restriction of the density behavior. One can obtain the above relation straightforward by doing analogous considerations as with respect to the finiteness of halo mass resulting from the condition (1). These considerations lead to a restriction for the introduced parameter $\epsilon$, namely it must be $\epsilon>1 / 2$, which exactly corresponds to the above obtained relation with respect to $m$.

The expression (20) for $\rho$ indicates that at radii significantly apart from zero $\left(\Phi / C_{0}^{1 /(1-\kappa)}\right.$ is not longer small) the density falls off more steeply than the obtained power asymptotes (see Fig. 2). This may explain that the asymptotic exponent $m=1 / 2$ is not as steep as the exponents found in $N$-body simulations. The latter ones can be obtained only at radii still sufficiently far away from $r=0$.

We have to stress, that the obtained asymptote (22) at $r \rightarrow 0$ is the solution if supposing a power-law behavior for $\Phi$ with 


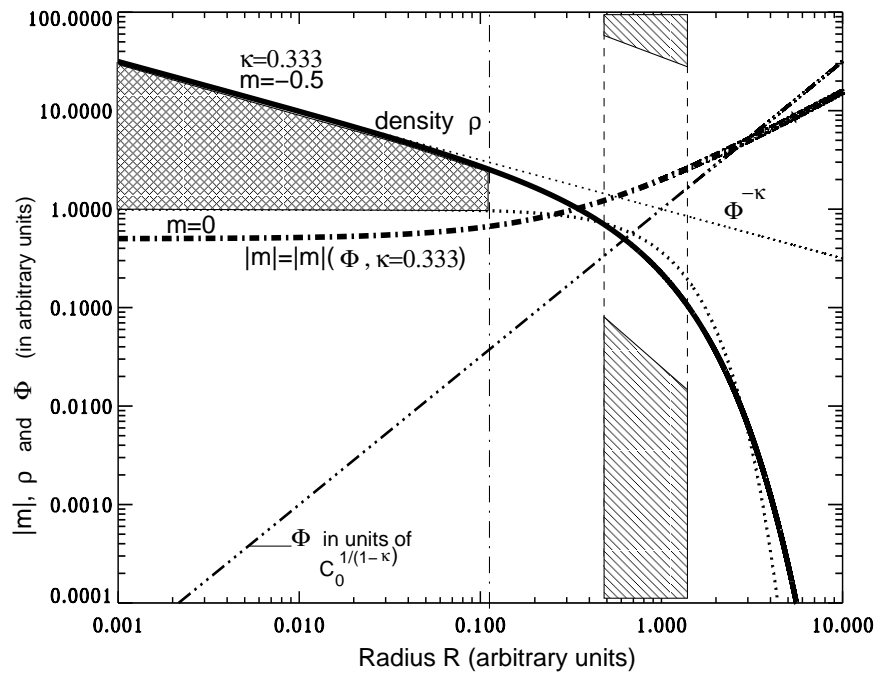

Fig. 2. The figure shows the range of possible density asymptotes at $r \rightarrow 0$ (upper left shaded area). This area is bordered by the limit case asymptote with $(\kappa=1 / 3, m=1 / 2)$ and the asymptote $(\kappa=0, m=0)$. The dashed dotted vertical line denotes the radii up to which the approximation $\rho \propto \Phi^{-\kappa}$ reliably works. For larger radii up to the right shaded area the expression (20) must be taken into account. The thick solid and the dotted lines give the corresponding behavior. The thick dot-dashed line gives the behavior of the effective power index (the absolute value is plotted). Throughout the range of radii where $\Phi$ can still be considered as small the index $|m|$ is increasing and of order unity.

respect to $r$. If replacing the density $\rho$ in the Poisson equation by its expression (20) we obtain a highly nonlinear differential equation which posses also solutions leading to a non-singular behavior of the density at $r \rightarrow 0$ as given below.

The result of the above considerations can be expressed as follows: If one supposes a behavior of the quantity $\rho$ obeying a power law with respect to the radial coordinate $\rho \propto r^{-m}$ then the power index is restricted by $0 \leq m \leq 1 / 2$ at $r \approx 0$.

Note however, that even if we are not demanding that $\Phi$ is a power law with respect to $r$ the more general relation (19) still holds.

We now consider the case $\kappa=0$ which represents the possibility of a non-vanishing velocity dispersion at $r=0$. Taking into account in the Jeans equation for $\sigma^{2}$ only the linear contributions of $\Phi$ and $\mathrm{d} \Phi / \mathrm{d} r$ we obtain for the density

$\rho \propto\left(1+a_{1} \Phi\right)^{-\left(1+\frac{1}{C_{0} a_{1}}\right)}$.

From the Poisson equation we get then the asymptotes for $\Phi \propto$ $r^{2}$. According to Eq. (30) the density remains finite at $r \rightarrow 0$, in this case. The shape of the density asymptote in the vicinity of $r \rightarrow 0$ is determined by $a_{1}$ and $C_{0}$. E.g., for $a_{1}=1 / 3$ and $C_{0}=2$ the asymptotic density profile is the Plummer's law.

Note, for all cases where $N \geq 1$ coefficients in (18) vanish, i.e., $a_{i}=0, i=1, \ldots N$ we are led to the standard Emden form and the approximate solution describes an isothermal sphere. However the condition $\Phi(0)=0$ leads inevitably to $\Phi(r) \propto r^{2}$ at $r \rightarrow 0$. All those solutions lead to an asymptotic core with constant density. The larger the non-vanishing dispersion $\sigma_{0}$ at the centre is the faster the core solution will be attained.

\section{Summary and conclusions}

We have systematically investigated the asymptotic behavior of the quantities describing spherically symmetric relaxed dark matter halos. We have drawn particular attention to the behavior near the centre $r=0$. The most restrictive boundary condition we have used is $\Phi(0)=\mathrm{d} \Phi / \mathrm{d} r(0)=0$. This means that the dark matter is considered to be continuously distributed, i.e., the gravitational potential at $r=0$ remains finite and no singular point mass at the centre is allowed.

This is in agreement with the suppositions for deriving the Jeans equation from the collisionless Boltzmann equation. It is demanded that each particle is moving within the smooth potential of all other particles and that no close two-body encounters take place. The latter is certainly true for the real dark matter distribution. Within $N$-body simulations encounters cannot be entirely excluded. The probability for an encounter between two particles is proportional to the particle density. Thus especially near the dense halo centres infalling particles with small impact parameter may undergo "collisions" just within this region. Therefore, one may generally expect some deviation of the particle distribution in the halos formed during $N$-body simulations from the results given here where we assume that the suppositions for the Jeans equation are well satisfied.

The asymptotic behavior of the velocity dispersion as function of the potential $\Phi$ at small and large radii can be given by (18) and (2), respectively. Provided the asymptotic density profile at large $r$ is characterized by a power asymptote with respect to $r$ then the parameters entering the asymptotic relation between the velocity dispersion and the gravitational potential $\sigma_{\mathrm{r}}^{2}=\alpha\left(\Phi_{\infty}-\Phi\right)^{p}$ can be further restricted. For $p<1$ no power law asymptote does exist which fulfills the boundary condition $\rho \rightarrow 0$ at $r \rightarrow \infty$. For $p>1$ the density profile exhibits an exponentially fast decrease at large distances and the halo structure is given by the mass concentration within a finite radius. For the case $p=1$ the parameter $\alpha$ is restricted by the relation $1 /(4-2 \beta)<\alpha<1 / 2$. This is in agreement with the observed velocity dispersion profiles.

We get at small radii an asymptotic behavior which seems to differ from the behavior derived from current results of numerical simulations. Assuming a power law behavior of the density profile $\rho(r) \propto r^{-m}$ at $r \rightarrow 0$ our analysis predicts $m<1$ for all cases being in agreement with the initial conditions. If we demand that the gradient for the pressure analog $\rho \sigma^{2}$ should vanish at $r=0$, then this leads to the even more restrictive relation $m<1 / 2$. Thus, if at small radii where $\Phi \ll\left(C_{0}(1-\kappa)\right)^{1 /(1-\kappa)}$ is valid, a power asymptote $\rho(r) \propto r^{-m}$ exists then $m$ must obey the above restrictions, i.e., a power asymptote according to the NFW profile cannot be continued to the innermost region under the assumption that the halo can be described by the Jeans equation.

While an analytic behavior of $\sigma^{2}$ with respect to $\Phi$ at $\Phi \rightarrow$ 0 can be well justified, the existence of a power asymptote with respect to $r$ for all the quantities is not mandatory. In particular, postulating the existence of a power asymptote for the density with respect to $r$ might be a too strong requirement. All solutions with nonvanishing velocity dispersion at the very centre lead asymptotically to a core with $\rho(0)=\rho_{0}<\infty$. 
We see two reasons that our results seem not to be in agreement with the results of the numerical simulations.

(i) The resolution of the available numerical simulations is probably not yet high enough to resolve the region $\Phi \ll\left(C_{0}(1-\kappa)\right)^{1 /(1-\kappa)}$.

However, as can be seen from the relation (20) the effective power index becomes steeper with increasing radii.

This is illustrated by Fig. 2, where the absolute value of the effective power index $|m|$ is shown to be increasing to values of about and larger than unity although it is still $\Phi / C_{0}^{1 /(1-\kappa)}<1$. At $\Phi \geq 1$ the approximation is not longer valid. Thus the obtained density asymptote approximates the NFW profile at the low-radius-end. This would explain why the numerical results obtained for radii where $\Phi^{1-\kappa} / C_{0} /(1-\kappa) \ll 1$ is not longer valid lead to a much steeper profile $(m>1)$.

(ii) An other reason could be that the halos having formed within the simulations are not yet sufficiently isolated from their surroundings. Therefore, a description by the steady state Jeans equation may be partly questionable.

Mergers and further matter inflow may alter the physical situation essentially and can lead to a steepening of the inner density profile Dekel et al. (2002).

On the other hand a couple of observations seem to support strongly the existence of an asymptotic density profile at $r \rightarrow 0$ being much less steep than $m=1$, e.g., de Blok et al. (2001).

This may indicate that the inner density profile near the centre depends on whether or not the considered halo is interacting with the surroundings and on the way this happens.

Acknowledgements. We would like to thank Walter Dehnen for useful comments on the manuscript. We thank the anonymous referee for his critical remarks and useful suggestions.

\section{References}

Binney, J., \& Tremaine, S. 1987, Galactic dynamics (Princeton, NJ: Princeton University Press, 1987, 747 p.)

Binney, J. J., \& Evans, N. W. 2001, MNRAS, 327, L27

Crone, M. M., Evrard, A. E., \& Richstone, D. O. 1994, ApJ, 434, 402 de Blok, W. J. G., \& McGaugh, S. S. 1996, ApJ, 469, L89

de Blok, W. J. G., McGaugh, S. S., \& Rubin, V. C. 2001, AJ, 122, 2396
Dekel, A., Devor, J., \& Arad, I. 2002, Galactic Halo Cusp versus Core: Tidal Effects in Mergers, in A New Era In Cosmology, ASP Conf. Ser., ed. T. Shanks, \& N. Metcalf

Dubinski, J., \& Carlberg, R. G. 1991, ApJ, 378, 496

Eke, V. R., Navarro, J. F., \& Frenk, C. S. 1998, ApJ, 503, 569

El-Zant, A., Shlosman, I., \& Hoffman, Y. 2001, ApJ, 560, 636

Frenk, C. S., White, S. D. M., Davis, M., \& Efstathiou, G. 1988, ApJ, 327, 507

Fukushige, T., \& Makino, J. 2001, ApJ, 557, 533

Ghigna, S., Moore, B., Governato, F., et al. 1998, MNRAS, 300, 146

Ghigna, S., Moore, B., Governato, F., et al. 2000, ApJ, 544, 616

Hoeft, M., Mücket, J. P., \& Gottlöber, S. 2003, submitted

Iliev, I. T., \& Shapiro, P. R. 2001, MNRAS, 325, 468

Jing, Y. P., \& Suto, Y. 2000, ApJ, 529, L69

Klypin, A., Kravtsov, A. V., Bullock, J. S., \& Primack, J. R. 2001, ApJ, 554, 903

Kravtsov, A. V., Klypin, A. A., \& Khokhlov, A. M. 1997, ApJS, 111, 73

Łokas, E. L., \& Hoffman, Y. 2000, ApJ, 542, L139

McGaugh, S. S., \& de Blok, W. J. G. 1998, ApJ, 499, 66

Moore, B., Governato, F., Quinn, T., Stadel, J., \& Lake, G. 1998, ApJ, 499, L5

Moore, B., Quinn, T., Governato, F., Stadel, J., \& Lake, G. 1999, MNRAS, 310, 1147

Milgrom, M. 1997, ApJ, 476, 22

Navarro, J. F. 2001, in Invited review presented at IAU Symp. 208, Astrophysical SuperComputing using Particles, ed. J. Makino, \& P. Hut, 10680

Navarro, J. F., Frenk, C. S., \& White, S. D. M. 1996, ApJ, 462, 563

Navarro, J. F., Frenk, C. S., \& White, S. D. M. 1997, ApJ, 490, 493

Nusser, A., \& Sheth, R. K. 1999, MNRAS, 303, 685

Power, C., Navarro, J. F., Jenkins, A. et al. 2003, MNRAS, 338, 14

Quinn, P. J., Salmon, J. K., \& Zurek, W. H. 1986, Nature, 322, 329

Sellwood, J. A. 2003, ApJ, 587, 638

Shapiro, P. R., Iliev, I. T., \& Raga, A. C. 1999, MNRAS, 307, 203

Springel, V., Yoshida, N., \& White, S. D. M. 2001, New Astron., 6, 79

Syer, D., \& White, S. D. M. 1998, MNRAS, 293, 337

Taylor, J. E., \& Navarro, J. F. 2001, ApJ, 563, 483

Thomas, P. A., Colberg, J. M., Couchman, H. M. P., et al. 1998, MNRAS, 296, 1061

Weinberg, M. D., \& Katz, N. 2002, ApJ, 580, 627

van den Bosch, F. C., \& Swaters, R. A. 2001, MNRAS, 325, 1017

Verheijen, M. A. W. 1997, Ph.D. Thesis, University of Groningen 